\title{
Packing of wet monodisperse spheres
}

\author{
Zhongzheng Wang \\ School of Civil Engineering, The University of Sydney, NSW 2006, Australia and \\ Navier, Ecole des Ponts, Univ Gustave Eiffel, CNRS, Marne-la-Vallée, France \\ Jean-Michel Pereira \\ Navier, Ecole des Ponts, Univ Gustave Eiffel, CNRS, Marne-la-Vallée, France \\ Yixiang Gan* \\ School of Civil Engineering, The University of Sydney, NSW 2006, Australia
}

\begin{abstract}
We experimentally investigated the packing of wet monodisperse spheres with controlled falling height. The packing fraction are found to decrease with smaller grain size and free fall heights. A model describing the effects of interparticle force and falling height on packing fraction is developed by introducing a dimensionless length scale, representing the extent of particle rearrangement towards a denser state due to impacts of falling grains. A universal law is observed for both wet particles where capillary forces dominate and dry powders where van der Waals forces govern the packing behaviour. This study deepens the understanding of packing of cohesive spheres and provide a simple experimental method for generating granular media with tailored packing fraction.
\end{abstract}

Keywords: granular packing, monodisperse spheres, capillary force, cohesive granular media

\section{INTRODUCTION}

The random packing of granular materials has been subjected to extensive studies due to its various industrial applications and importance for the understanding of many fundamental physical problems [1-6]. The packing fraction of monodisperse cohesionless frictional particles ranges from $\sim 0.55$ (random very loose packing) to $\sim 0.64$ (random close packing), depending on the packing method and particle friction coefficient [7-16]. For frictionless particles, O'Hern et al. showed narrowed jamming threshold as the system size increases, approaching 0.648 in 3D, close to random close packing $[14,15]$. Moreover, studies on the effects of tapping on system of monodisperse spheres attribute the increase of packing fraction to the propagation of ordered packing structure from the boundary [17-19]. On the other hand, the packing fraction is found to decrease with smaller effective gravity or greater friction coefficient $[7,12,16]$. Further, Farrell et al. experimentally demonstrated how Stokes number controls the approach to the loose packing limit by varying the particle and fluid properties [10]. In the context of statistical mechanics, Ciamarra and Coniglio showed that the granular entropy, which reflects the number of mechanically stable states of volume fraction of a granular assembly, vanishes at upper and lower bound of packing fractions, while reaching a maximum at random loose packing [11]. Their work provided the first-principle definition of the commonly observed random loose packing fraction.

For spheres with diameter typically less than $100 \mu \mathrm{m}$, the van der Waals force becomes significant. The attractive interparticle force starts to influence the packing fraction, which have been observed both numerically and experimentally [20-27]. Through imposing a controlled external magnetic field on iron spheres, Forsyth et al. demonstrated lower packing fraction with greater interparticle forces [28], and they concluded that the void fraction of spherical particles depends only on the ratio of interparticle cohesive force to particle weight. Their claim was further confirmed by DEM simulations of settling of fine particles [24, 26, 29]. In fact, the van der Waals force explains the formation of fragile low density configurations such as quicksand [23, 25], and packing fraction as low as $15 \%$ has been experimentally created using ballistic deposition [22].

For larger particles, the effect of van der Waals force on packing fraction becomes negligible. However, the addition of liquid strengthens the cohesive force due to formation of liquid bridges [5, 6, 30-32]. Feng and Yu observed an increase in porosity of mono-sized glass beads when grains are added with water, followed by a plateau regime where porosity is insensitive to liquid content. Finally, the porosity decreases when more water is added. Similar behaviours have been observed in bidisperse and polydisperse systems [34-36]. The origin of these three distinct regimes can be readily understood by the relationship between liquid content and capillary force in granular media. At very low liquid content (asperity or roughness regime [37]), the capillary force increases with liquid volume due to surface

\footnotetext{
* yixiang.gan@sydney.edu.au
} 


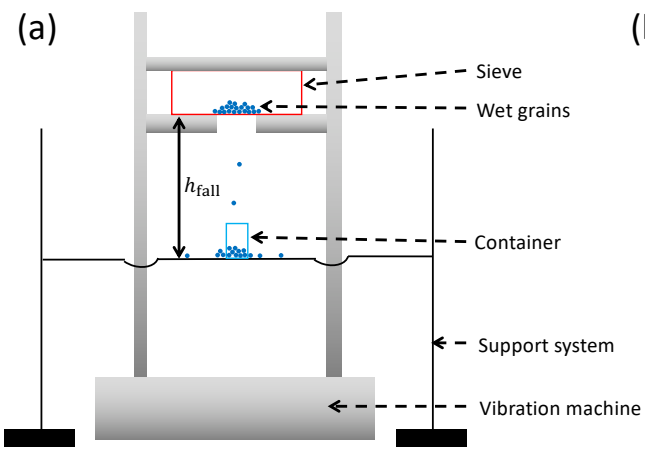

(b)

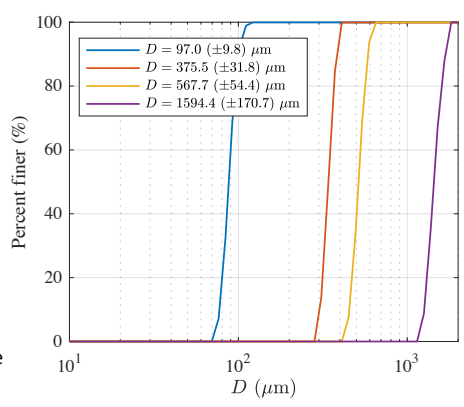

(c)

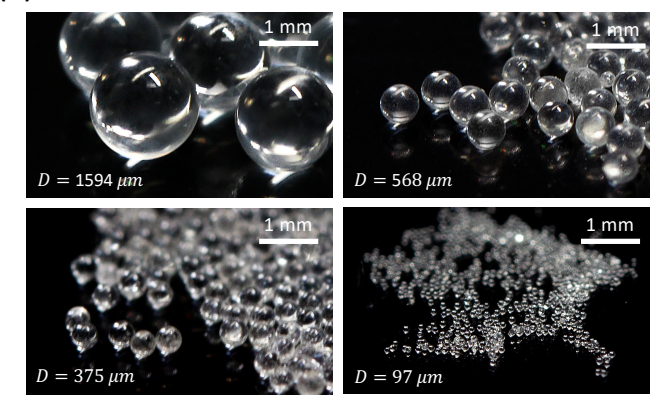

FIG. 1. (a) Schematic of the system for packing of wet spheres. (b) Cumulative grain size distribution. Values in bracket represent standard deviation. (c) Photos of glass beads with different sizes.

roughness. However, further increase in liquid content does not result in additional increase in interparticle force because the increase in liquid-solid contact area balances with decrease in Laplace pressure [30, 32, 37, 38]. This has been experimentally confirmed by measurements of the capillary force due to liquid bridge at grain scale and the tensile strength of wet granular pile at macroscopic scale $[5,39]$. Finally, menisci coalesce before the slurry state where liquid is in excess and no capillary effects exist [33, 40, 41].

The aforementioned studies of packing of wet cohesive spheres mostly focus on the effects of grain size, interparticle forces, and solid/liquid properties. Less attention has been paid in packing methods, given that most experiments were carried out by pouring grains into the container [27, 28, 33, 42, 43]. To systematically probe the influence of packing method on the packing state of granular materials, in this work, a simple experimental method for preparing granular media with desired packing fraction is presented. We investigate the effect of falling height $h_{\text {fall }}$ on packing fraction of wet monodisperse spherical glass beads with different grain sizes. A previously proposed relation describing the packing fraction $[20,21,24]$ is extended by introducing a dimensionless length scale, representing the degree of compaction due to gravity, where the effects of falling height can be captured.

\section{EXPERIMENTS}

Glass beads with a density of $2460 \mathrm{~kg} / \mathrm{m}^{3}$ [44] were mixed with water at an initial volumetric water content $w_{0}=5 \pm 0.1 \%$ to ensure the granular medium is in pendular state and liquid bridges are formed [5, 31, 44]. Then, the wet grains are placed in a sieve, which was shaked by a vibration machine (Impact SV008 Electromagnetic Sieve Shaker, frequency $50 \mathrm{~Hz}$ and intensity $3.3 \mathrm{~mm}$ ). A cylindrical container with inner diameter $13.70 \pm 0.01 \mathrm{~mm}$ and inner height $44.94 \pm 0.12 \mathrm{~mm}$ is placed $h_{\text {fall }}$ away under the sieve, being supported by struts that are disconnected with the vibration machine. Fig. 1(a) shows the schematic of the experiment setup. The same equipment has been used in a previous work by Than et al. [44]. Note that in order to ensure a constant $h_{\text {fall }}$ during the experiments, ideally, the supporting system should move downwards such that the surface of the grains stays at the same level as more grains fall into the container. However, we did not aim to produce such equipment due to extra complexity involved. Once the container is full, the grains in excess were carefully removed using a sharp plate, and medium was weighed before and after drying in an oven overnight at $105{ }^{\circ} \mathrm{C}$. The sieve grid size $\{0.2,1,1.5,3.15\}$ mm were used for glass beads with average diameters $\{97,375,568,1594\} \mu \mathrm{m}$, respectively (Fig. 1(c)). These sizes allow passing for maximum of two to three spheres and avoid clusters of grains (namely, aggregates or agglomerates, which leads to less homogeneous packing structure) $[40,43,45]$. The cumulative grain size distribution determined by laser diffraction technique (using Beckman Coulter Vsm+ Ls Variable Speed Fluid Module Plus) is shown in Fig. 1(b). Note that the data for packing of $97 \mu \mathrm{m}$ wet grains are obtained from Than et al. [44]. For packing of dry grains, given relative large size of glass beads (generally larger than $100 \mu \mathrm{m}$ ), the van der Waals force is not expected to significantly affect the packing fraction $[26,29,46]$. Therefore, the system is simply packed by the pouring method. To ensure there is no formation of liquid bridges at lab condition due to capillary condensation, the water contents at "dry" condition were measured to be less than $0.03 \%$, being smaller than $0.07 \%$, at which the liquid bridges start to form [31]. 


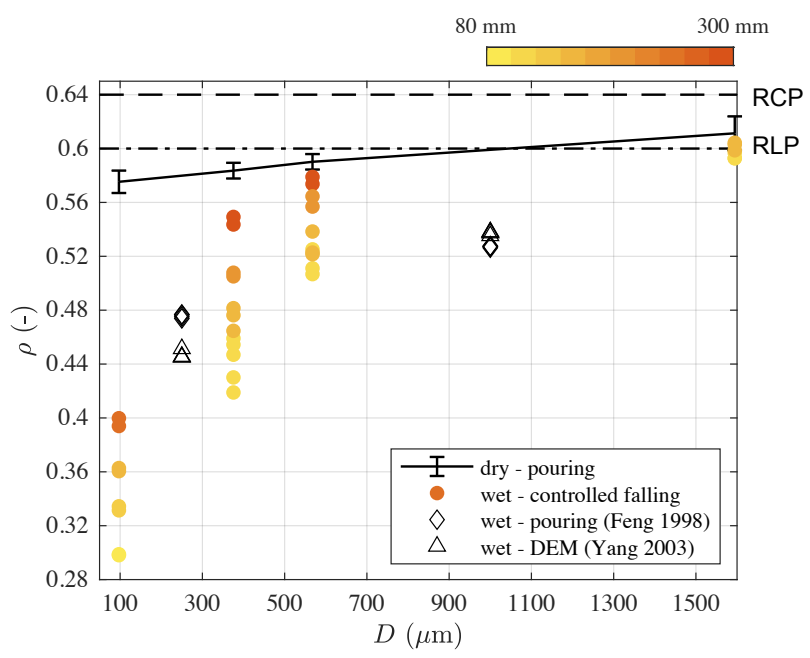

FIG. 2. Packing fraction $\rho$ of dry (solid line with errorbar) or wet (circles) monodisperse glass beads. The errorbar represents the standard deviation of 6 measurements. The colorbar represents different free fall height $h_{\text {fall }}$. Data from literature are added for comparison (experiments using pouring method [33] and DEM simulation [40]). The packing fraction normally observed at random close packing (RCP) and random loose packing (RLP) are marked by dashed line and dash-dot line, respectively.

(a)

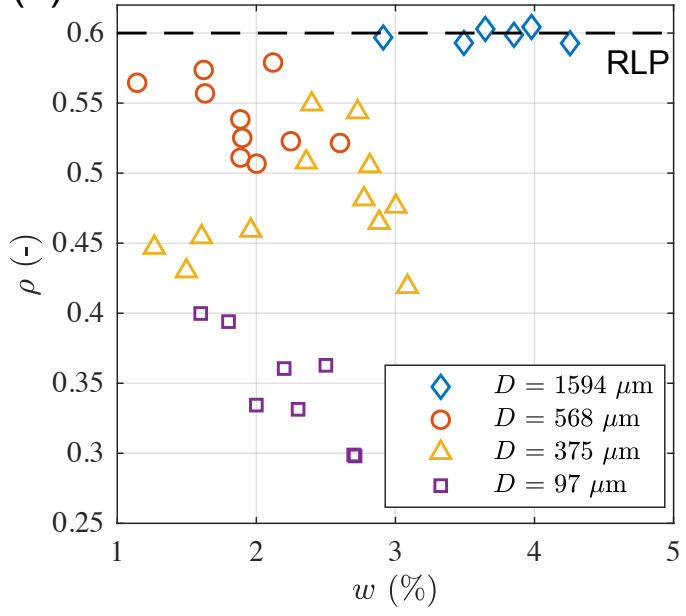

(b)

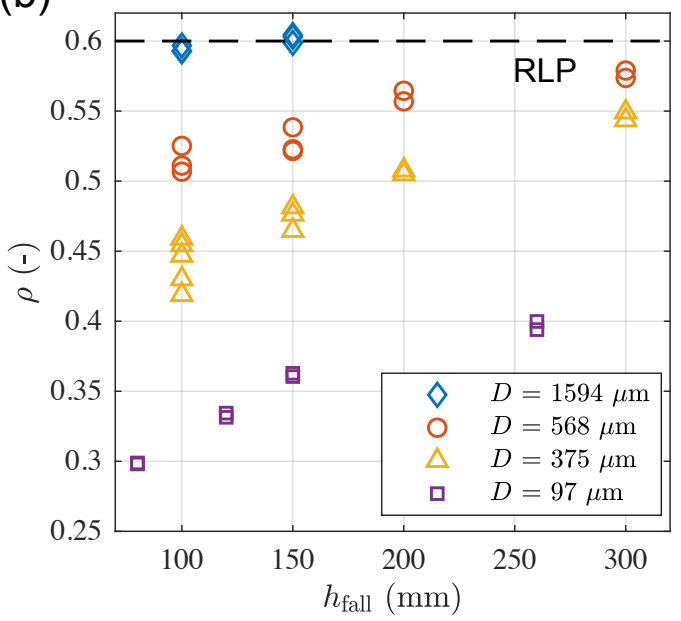

FIG. 3. (a) The influence of volumetric water content $w$ on packing fraction is not significant. (b) Packing fraction $\rho$ increases with larger height of free fall $h_{\text {fall }}$ for different grain sizes.

\section{RESULTS AND DISCUSSION}

Fig. 2 shows the packing fraction $\rho$ of monodisperse glass beads under dry or wet condition. For dry grains, the packing fraction is close to random loose packing of 0.6, consistent with past observations [33]. There is a slight decrease in $\rho$ for smaller grains. One may argue this could result from the boundary effects [36, 47]. Nevertheless, in the study of packing of cohesionless spheres in cylindrical containers by Zou and Yu [47], both the side-wall effect and top-bottom-wall effect (or thickness effect) lead to decrease in packing fraction for smaller system domain (corresponding to larger grains with the same container), which contradicts the trend in our results. Therefore, the decrease in packing fraction for smaller grains, despite small magnitude, is likely due to van der Waals force [20, 26, 33]. The packing fraction for wet grains is generally smaller than dry condition, and this decrease is more significant for smaller spheres. In addition, the colorbar shows the effects of free fall height $h_{\text {fall }}$ on $\rho$, with denser packing at greater $h_{\text {fall. }}$.

Previous studies have demonstrated increases in $\rho$ with higher liquid content at very low liquid content regime (typically less than $1 \sim 2 \%$ depending on grain size distribution) [33, 36, 42, 43]. However, Fig. 3(a) shows the relationship between $\rho$ and $w$ for all grain sizes, where no significant correlation between $w$ and $\rho$ can be seen. As 
discussed before, this is because the medium is in pendular state, and the capillary force due to liquid bridges is not sensitive to variations in water content within this regime $[5,33,39]$. By rearranging the dataset, Fig. 3(b) more quantitatively shows $\rho$ as a function of $h_{\text {fall }}$. It can be observed that $\rho$ increases monotonically with $h_{\text {fall }}$, ranging from around 0.3 to random loose packing of 0.6 marked by black-dashed line. Although $\rho$ increases with $h_{\text {fall }}$ for the investigated range of $h_{\text {fall }}$, it is expected the packing fraction will eventually reach a plateau as $h_{\text {fall }}$ further increases due to either (1) the particle reaches the terminal velocity due to air drag and the kinetic energy is saturated, or (2) the packing fraction approaches 0.6 and the system starts to jam. For $D=1594 \mu \mathrm{m}$ grains, the packing fraction is about 0.6 at the smallest $h_{\text {fall }}$; whilst for larger $h_{\text {fall }}>150 \mathrm{~mm}$, bounces were observed and the container could not be completely filled. However, even with greater $h_{\text {fall }}$ it is expected that the packing fraction will not surpass the value at dry condition as particles are already jammed.

To quantify the interplay between gravity and interparticle forces on packing fraction, a model has previously been proposed for fine particles [24, 29]:

$$
\rho=\rho_{0}\left(1-e^{a \cdot r^{b}}\right)
$$

where $\rho_{0}$ is the packing fraction without the presence of attractive interparticle force with values between random loose packing (0.6) and random close packing (0.64), $a$ and $b$ are fitting parameters, and $r$ is the "force ratio" of the magnitudes of interparticle force to the gravity. However, Eqn. (1) does not consider packing methods and will produce a single value of $\rho$ for any given grain size with different $h_{\text {fall }}$. In order to take consideration of the free fall height, an "energy ratio" $r_{\mathrm{E}}$ can be defined as:

$$
r_{\mathrm{E}}=\frac{F_{\text {inter }} \cdot l}{E_{\mathrm{k}}},
$$

where $F_{\text {inter }}$ is the interparticle force, $l$ is the characteristic length, representing the displacement during the process of particles rearrangement, and $E_{\mathrm{k}}$ is the kinetic energy upon impact, which can be approximated by the gravitational potential energy. Note that $r_{\mathrm{E}}$ represents the proportion of kinetic energy dissipated due to interparticle forces (e.g., through enhanced frictional force from liquid bridges). Therefore, $r_{\mathrm{E}}$ approaches to unity when the interparticle cohesive forces are significant comparing to gravity. Previous studies have indicated that the ratio of interparticle force to gravity needs to be much larger than one (in the order of 10) in order for the interparticle force to have significant effect on packing fraction [20,29]. This can be explained by the fact that there is no preferred direction in the interparticle force due to random arrangement of grains, unlike gravity [20]. For wet spheres, the capillary force $F_{\mathrm{c}}$ due to liquid bridges can be calculated as $F_{c}=2 \pi \gamma R \cos \theta[30,32,37]$, where $\gamma$ is the surface tension, $R$ is the radius, and $\theta$ is the contact angle. Thus, for the force ratio $\frac{F_{c}}{F_{g}}=\frac{3 \gamma \cos \theta}{2 R^{2} \rho g} \approx 10$ with the average contact angle between glass beads and water $\theta \approx 60^{\circ}[48]$ and density of $2460 \mathrm{~kg}^{3} / \mathrm{m}$, an approximate threshold grain size can be obtained as $D^{*}=\sqrt{\frac{6 \gamma \cos \theta}{10 \cdot \rho g}} \approx 950 \mu \mathrm{m}$. For grain sizes larger than $D^{*}, r_{E} \ll 1$, and it is expected that the packing fraction is close to random loose packing 0.6, insensitive to free fall height, which is confirmed by Fig. 3(b). For grain sizes less than $D^{*}, r_{E}$ approaches to 1 . Under this regime, a dimensionless length scale can be introduced $l^{*}=l / D$, which reflects the relative displacement during the process of particle rearrangement. Then, with Eqn. (2), assuming $r_{E}=1$ for simplicity, one obtains:

$$
l^{*}=\frac{E_{\mathrm{k}}}{F_{\text {inter }} \cdot D} .
$$

Therefore, $l^{*}$ can be regarded as the the degree of compaction due to gravity, i.e., the extent of particle rearrangement towards a denser state from the packing state of diffusion limited aggregation. Either smaller $E_{\mathrm{k}}$ or greater $F_{\text {inter }}$ lead to decrease in $l^{*}$, implying less compaction and thus looser packing state. It is worth mentioning that, for the case of wet particles with negligible van der Waals force where the interparticle force is mainly from capillary force, $l^{*}$ can be thought as a generalization of Weber number $l^{*}=\frac{\rho D v^{2}}{12 \gamma \cos \theta}=\frac{\text { We }}{12 \cos \theta}$, which provides a measure of relative importance of inertia compared to surface tension. Fig. 4 plots the packing fraction as a function of $l^{*}$. Collapse of data can be observed for different grain sizes and free fall heights. For grain size larger than $D^{*}$, as mentioned before the forces from liquid bridge will not be the major source of energy dissipation, i.e, $r_{E} \ll 1$. Therefore, $r_{E}=0.1$ is used as an estimation for $D=1594 \mu \mathrm{m}$. Also, the model from Eqn. (1) with $l^{*}$ replacing $r$ are plotted with solid line with fitted $a=-19.2, b=0.478$ and $\rho_{0}=0.602$.

We also check the validity of the model for describing dry grains with diameter less than $100 \mu \mathrm{m}$ where van der Waals force plays an important role. The attractive van der Waals force between two particles can be calculated by $[20,21,29]$ :

$$
F_{v}=\frac{A}{6} \frac{64 R_{i}^{3} R_{j}^{3}\left(s+R_{i}+R_{j}\right)}{\left(s^{2}+2 R_{i} s+2 R_{j} s\right)^{2}\left(s^{2}+2 R_{i} s+2 R_{j} s+4 R_{i} R_{j}\right)^{2}},
$$




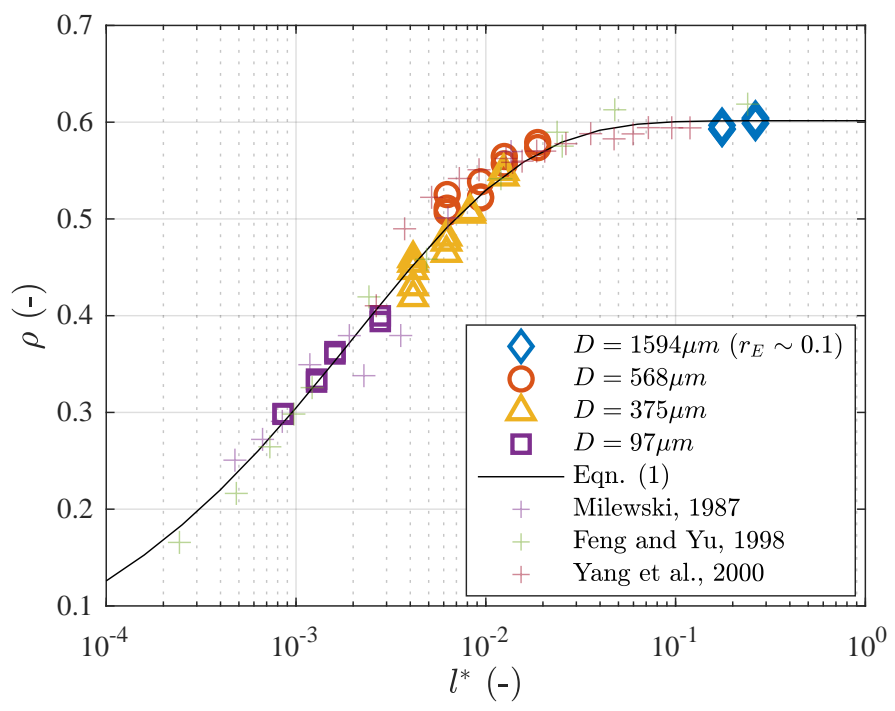

FIG. 4. Packing fraction $\rho$ of wet spheres as a function of $l^{*}$ for grains of different sizes and $h_{\mathrm{fall}}$. Solid line is from Eqn. (1) with $r$ replaced by $l^{*}$. Data from literature for dry powders are added for comparison where the interparticle force is calculated by Eqn.4.

where $R_{i}, R_{j}$ are radii of the two spheres, $\mathrm{A}$ is the Hamaker constant that is related to material properties, being $6.5 \times 10^{-20} \mathrm{~J}$ for glass beads, and $s$ is the separation distance with typical value of $1 \times 10^{-10} \mathrm{~m}[1,21]$. With monodisperse spheres and assuming the separation distance is much less than the grain radius, Eqn. (4) reduces to $F_{v}=A R /\left(12 s^{2}\right)$. Data from literature of packing of fine powders are shown as "+" signs in Fig. 4. Since we did not find the corresponding falling height for the data, the $h_{\text {fall }}$ is fitted to be $5 \mathrm{~mm}$. However, this value is likely to be smaller than the typical $h_{\text {fall }}$ in laboratory conditions. One of the sources of the error could be from the calculation in interparticle forces, especially when the accurate estimation of the separation distance $s$ can be difficult. This error will lead to a shift of data in the horizontal direction in Fig. 4. Nevertheless, the general shape of the data from literature, which is independent of $h_{\text {fall }}$ or $s$, matches well with the proposed theory.

\section{CONCLUSIONS}

The packing of wet monodisperse spheres was experimentally studied. Glass beads with different sizes were packed under controlled falling height. It is observed that both grain size and free fall height significantly impact the packing fraction. A dimensionless length scale is introduced, which reflects the extent of particle rearrangement towards a denser state due to impacts from falling grains, extending the existing model for describing the packing fraction of cohesive spheres, where the effect of falling heights can be well captured. Based on the proposed theory, collapses of data are observed for both wet particles where capillary force dominates and dry powders where van der Waals force is essential. Our study deepens the understanding of packing of cohesive spheres and provide a simple experimental method for preparing granular media with desired packing fraction by using the proposed universal law.

\section{ACKNOWLEDGMENTS}

This work was financially supported by Australian Research Council (Projects DP170102886) and The University of Sydney SOAR Fellowship. YG acknowledges the financial support of Labex MMCD(ANR-11-LABX-022-01) for his stay at Laboratoire Navier at ENPC. ZW thanks Baptiste Chabot, Xavier Boulay, Loïc Lesueur, and Emmanuel De Laure for assistance on experiments.

[1] H. Zhu, Z. Zhou, R. Yang, and A. Yu, Discrete particle simulation of particulate systems: Theoretical developments, Chemical Engineering Science 62, 3378 (2007), frontier of Chemical Engineering - Multi-scale Bridge between Reductionism 
and Holism.

[2] H. Zhu, Z. Zhou, R. Yang, and A. Yu, Discrete particle simulation of particulate systems: A review of major applications and findings, Chemical Engineering Science 63, 5728 (2008).

[3] H. Chen, W. Liu, and S. Li, Random loose packing of small particles with liquid cohesion, AIChE Journal 65, 500 (2019), https://aiche.onlinelibrary.wiley.com/doi/pdf/10.1002/aic.16440.

[4] S. Chen, W. Liu, and S. Li, A fast adhesive discrete element method for random packings of fine particles, Chemical Engineering Science 193, 336 (2019).

[5] M. Scheel, R. Seemann, M. Brinkmann, M. Di Michiel, A. Sheppard, B. Breidenbach, and S. Herminghaus, Morphological clues to wet granular pilestability, Nature materials 7, 189 (2008).

[6] M. Scheel, R. Seemann, M. Brinkmann, M. D. Michiel, A. Sheppard, and S. Herminghaus, Liquid distribution and cohesion in wet granular assemblies beyond the capillary bridge regime, Journal of Physics: Condensed Matter 20, 494236 (2008).

[7] G. Y. Onoda and E. G. Liniger, Random loose packings of uniform spheres and the dilatancy onset, Phys. Rev. Lett. 64, 2727 (1990).

[8] L. Liu, Z. Zhang, and A. Yu, Dynamic simulation of the centripetal packing of mono-sized spheres, Physica A: Statistical Mechanics and its Applications 268, 433 (1999).

[9] X. Cheng, Experimental study of the jamming transition at zero temperature, Phys. Rev. E 81, 031301 (2010).

[10] G. R. Farrell, K. M. Martini, and N. Menon, Loose packings of frictional spheres, Soft Matter 6, 2925 (2010).

[11] M. P. Ciamarra and A. Coniglio, Random very loose packings, Phys. Rev. Lett. 101, 128001 (2008).

[12] M. Jerkins, M. Schröter, H. L. Swinney, T. J. Senden, M. Saadatfar, and T. Aste, Onset of mechanical stability in random packings of frictional spheres, Phys. Rev. Lett. 101, 018301 (2008).

[13] A. J. Liu and S. R. Nagel, The jamming transition and the marginally jammed solid, Annual Review of Condensed Matter Physics 1, 347 (2010).

[14] C. S. O'Hern, S. A. Langer, A. J. Liu, and S. R. Nagel, Random packings of frictionless particles, Phys. Rev. Lett. 88, 075507 (2002).

[15] C. S. O'Hern, L. E. Silbert, A. J. Liu, and S. R. Nagel, Jamming at zero temperature and zero applied stress: The epitome of disorder, Phys. Rev. E 68, 011306 (2003).

[16] L. E. Silbert, Jamming of frictional spheres and random loose packing, Soft Matter 6, 2918 (2010).

[17] A. D. Rosato, O. Dybenko, D. J. Horntrop, V. Ratnaswamy, and L. Kondic, Microstructure evolution in density relaxation by tapping, Phys. Rev. E 81, 061301 (2010).

[18] V. Ratnaswamy, A. D. Rosato, D. Blackmore, X. Tricoche, N. Ching, and L. Zuo, Evolution of solids fraction surfaces in tapping: simulation and dynamical systems analysis, Granular Matter 14, 163 (2012).

[19] W. Dai, J. Reimann, D. Hanaor, C. Ferrero, and Y. Gan, Modes of wall induced granular crystallisation in vibrational packing, Granular Matter 21, 26 (2019).

[20] R. Y. Yang, R. P. Zou, and A. B. Yu, Computer simulation of the packing of fine particles, Phys. Rev. E 62, 3900 (2000).

[21] A. Yu, C. Feng, R. Zou, and R. Yang, On the relationship between porosity and interparticle forces, Powder Technology 130, 70 (2003).

[22] J. Blum and R. Schräpler, Structure and mechanical properties of high-porosity macroscopic agglomerates formed by random ballistic deposition, Phys. Rev. Lett. 93, 115503 (2004).

[23] D. Lohse, R. Rauhé, R. Bergmann, and D. Meer, Creating a dry variety of quicksand, Nature 432, 689 (2005).

[24] K. J. Dong, R. Y. Yang, R. P. Zou, and A. B. Yu, Role of interparticle forces in the formation of random loose packing, Phys. Rev. Lett. 96, 145505 (2006).

[25] P. B. Umbanhowar and D. I. Goldman, Low density fragile states in cohesive powders, American Journal of Physics 74, $720(2006)$.

[26] R. Yang, R. Zou, K. Dong, X. An, and A. Yu, Simulation of the packing of cohesive particles, Computer Physics Communications 177, 206 (2007), proceedings of the Conference on Computational Physics 2006.

[27] E. Parteli, J. Schmidt, C. Blümel, K.-E. Wirth, W. Peukert, and T. Pöschel, Attractive particle interaction forces and packing density of fine glass powders, Scientific reports 4, 6227 (2014).

[28] A. J. Forsyth, S. R. Hutton, C. F. Osborne, and M. J. Rhodes, Effects of interparticle force on the packing of spherical granular material, Phys. Rev. Lett. 87, 244301 (2001).

[29] K. J. Dong, R. Y. Yang, R. P. Zou, and A. B. Yu, Settling of particles in liquids: Effects of material properties, AIChE Journal 58, 1409 (2012).

[30] Y. I. Rabinovich, M. S. Esayanur, and B. M. Moudgil, Capillary forces between two spheres with a fixed volume liquid bridge: Theory and experiment, Langmuir 21, 10992 (2005), pMID: 16285763.

[31] Z. Fournier, D. Geromichalos, S. Herminghaus, M. M. Kohonen, F. Mugele, M. Scheel, M. Schulz, B. Schulz, C. Schier, R. Seemann, and A. Skudelny, Mechanical properties of wet granular materials, Journal of Physics: Condensed Matter 17, S477 (2005).

[32] H.-J. Butt and M. Kappl, Normal capillary forces, Advances in Colloid and Interface Science 146, 48 (2009).

[33] C. Feng and A. Yu, Effect of liquid addition on the packing of mono-sized coarse spheres, Powder Technology 99, 22 (1998).

[34] R.-P. Zou, C.-L. Feng, and A.-B. Yu, Packing density of binary mixtures of wet spheres, Journal of the American Ceramic Society 84, 504 (2001), https://ceramics.onlinelibrary.wiley.com/doi/pdf/10.1111/j.1151-2916.2001.tb00690.x.

[35] R. Zou, J. Xu, C. Feng, A. Yu, S. Johnston, and N. Standish, Packing of multi-sized mixtures of wet coarse spheres, Powder Technology 130, 77 (2003). 
[36] J.-F. Bruchon, Investigation by means of X-ray computed tomography of capillary collapse in granular materials, Theses, Université Paris-Est (2014).

[37] T. C. Halsey and A. J. Levine, How sandcastles fall, Phys. Rev. Lett. 80, 3141 (1998).

[38] H.-J. Butt, Capillary forces: Influence of roughness and heterogeneity, Langmuir 24, 4715 (2008), pMID: 18442225.

[39] B. Mielniczuk, T. Hueckel, and M. S. E. Youssoufi, Evaporation-induced evolution of the capillary force between two grains, Granular Matter 16, 815 (2014).

[40] R. Y. Yang, R. P. Zou, and A. B. Yu, Numerical study of the packing of wet coarse uniform spheres, AIChE Journal 49, 1656 (2003).

[41] N. Mitarai and F. Nori, Wet granular materials, Advances in Physics 55, 1 (2006).

[42] C. Feng and A. Yu, Quantification of the relationship between porosity and interparticle forces for the packing of wet uniform spheres, Journal of Colloid and Interface Science 231, 136 (2000).

[43] J. Q. Xu, R. P. Zou, and A. B. Yu, Packing structure of cohesive spheres, Phys. Rev. E 69, 032301 (2004).

[44] V.-D. Than, P. Aimedieu, J.-M. Pereira, J.-N. Roux, M. Bornert, and A.-M. Tang, Macro-microscopic one-dimensional compression of wet granular soils by experimental investigation, E3S Web of Conferences 9, 06001 (2016).

[45] J.-F. Bruchon, J.-M. Pereira, M. Vandamme, N. Lenoir, P. Delage, and M. Bornert, Full 3d investigation and characterisation of capillary collapse of a loose unsaturated sand using x-ray ct, Granular Matter 15, 783 (2013).

[46] A. Yu, J. Bridgwater, and A. Burbidge, On the modelling of the packing of fine particles, Powder Technology 92, 185 (1997).

[47] R. Zou and A. Yu, The packing of spheres in a cylindrical container: the thickness effect, Chemical Engineering Science 50, 1504 (1995).

[48] K. A. Klise, D. Moriarty, H. Yoon, and Z. Karpyn, Automated contact angle estimation for three-dimensional x-ray microtomography data, Advances in Water Resources 95, 152 (2016), pore scale modeling and experiments. 